Revista

\title{
Multi-Ensayos
}

Vol. 6, núm. 12

ISSN: 2412-3285

https://multiensayos.unan.edu.ni

DOI: https://doi.org/10.5377/multiensayos.v6i12.10114

\section{Corrientes epistemológicas para construir conocimiento en Geografía y su relevancia para el estudio de la identidad territorial}

\section{Epistemological currents to build knowledge in Geography and its relevance for the study of territorial identity}

Samanta María Espinoza Rivera ${ }^{1}$

Recibido: 27 de febrero de 2020. Aceptado: 15 de junio de 2020

\section{RESUMEN}

El presente ensayo tiene el propósito es exponer un breve recorrido por las principales corrientes epistemológicas fundamentales en la construcción del conocimiento científico, en específico de la Geografía y su relevancia teórico para el estudio de la identidad territorial. Se parte del hipotético que las corrientes del conocimiento que a continuación se esbozaran son relevantes ya que contribuyen con enfoques teóricos para el tema de identidad territorial. El escrito desarrolla dos puntos, el primero describe las corrientes epistemológicas, iniciando con la contienda de los planteamientos sobre la ciencia, paralelo al desarrollo del pensamiento filosófico; dando énfasis en aquellas corrientes que incitaron el desarrollo de la ciencia geográfica. Es importante destacar que en principio existen dos enfoques bien marcados, el positivismo y el radical, este último le impregnó con fuerza el carácter sociocrítico a la Geografía. El segundo, considera las corrientes epistemológicas que tienen relevancia teórica y metodológica en la disertación de la identidad territorial. Posteriormente se presentan las consideraciones finales.

Palabras clave: corriente epistemológica; Geografía; identidad territorial.

\section{ABSTRACT}

The purpose of this essay is to present a brief overview of the main fundamental epistemological tests in the construction of scientific knowledge, in the specifications of Geography and their relevance theoretical for the study of territorial identity. It is assumed that the currents of knowledge outlined below are relevant as they are faced with theoretical approaches to the subject of territorial identity. The paper develops two points, the first describes epistemological currents, beginning with the contention of the approaches to science, parallel to the development of philosophical thought; emphasizing current currents that incited the development of geographic science. Foremost there are two well-marked approaches, positivism and radicalism, the latter strongly impregnated the socio-critical character of Geography. The second considers the epistemological currents that have theoretical and methodological relevance in the dissertation of territorial identity. In short, the aim is to identify the directions that allow reflection and knowledge. The final considerations are presented below.

Keywords: epistemological current; Geography; territorial identity.

1 Profesora del Departamento de Geografía. Universidad Nacional Autónoma de Nicaragua, Managua. Correo electrónico: samanta.espinoza@unan.edu.ni

(c) 2020 Revista Multi-Ensayos. 


\section{INTRODUCCIÓN}

¿A qué llamamos corriente epistemológica? En este sentido, la llamaré como un punto de vista acerca de la realidad, estudiando el conocimiento como un fenómeno, del porqué ocurren los hechos a nuestro alrededor y de cómo funcionan. Estas corrientes se suscitaron por la necesidad del ser humano en tener el control de lo desconocido y por consiguiente el poder.

En relación al párrafo anterior, es importante explicar el concepto de corriente epistemológica. Por ello, se expone el concepto de "corriente" y epistemología". Con relación al término "corrientes", estas son aquellas corrientes filosóficas que proceden de distintas agrupaciones de filósofos que definen según las características comunes y opiniones compartidas sobre filosofía. Estas se han desarrollado con el propósito de compartir y discutir diversos razonamientos lógicos y métodos sobre conceptos abstractos relacionados con la humanidad y el contexto que nos rodea. Por ello, cada una de las corrientes que existe responde a una época, un hecho histórico o surge de la necesidad de expresar contrariedad u oposición a una lógica en particular.

Por lo que se refiere a la "epistemología" se la define como la 'teoría del conocimiento'; etimológicamente, su raíz deriva del griego episteme, ciencia, pues por extensión se acepta que ella es la base de todo conocimiento. Su definición formal es el estudio crítico del desarrollo, métodos y resultados de las ciencias. Se la define también como el campo del saber que trata del estudio del conocimiento humano desde el punto de vista científico (Ramírez, 2009, pág. 217).

Ramírez (2009), señala que la epistemología busca responder incógnitas trascendentales: ¿Cómo se desarrollaron, y desarrollan, los modelos o las teorías sobre el pensamiento predominantes en cada época?, ¿Cómo avanza el conocimiento humano?, ¿Las teorías se complementan o compiten entre sí?, interrogaciones que adquieren mayor significado ahora cuando el ser humano con ímpetu surca, en la investigación científica y tiene la necesidad de formular interrogantes, indagar respuestas y facilitar técnicamente el conocimiento.

El conocimiento, tal como se le concibe hoy, es el proceso progresivo y gradual desarrollado por el hombre para aprehender su mundo y realizarse como individuo, y especie. El conocimiento desde el punto de vista epistemológico, es un proceso mental que da lugar al nacimiento de nuevas ideas.

Por consiguiente, las corrientes epistemológicas son aquellas corrientes y sistemas de pensamiento que conllevan abstracciones paralelas en la amanera de conocer el mundo. Cada una de estas formas particulares de interpretar el conocimiento tiene 'su' propia visión y por tanto un enfoque parcial para conocer en el contexto la generación y la solidez de su validación y aplicación, pues substancian el conocimiento desde su propia circunstancia. Estas se conciben la en forma ecléctica a partir del concepto de conocimiento obtenido de la ciencia y la filosofía.

Dicho lo anterior, el presente ensayo tiene el propósito de exponer las corrientes epistemológicas que han sido motores fundamentales en la generación del conocimiento científico. En este sentido, el desarrollo de la ciencia geográfica ha sido un producto de la influencia de distintas corrientes epistemológicas. Así que en el progreso de este escrito conoceremos las corrientes del pensamiento geográfico que se originaron en contextos de tiempo y espacio diferentes y que no están ajenas a la evolución de las ciencias como tal. 
El presente escrito aborda en primer lugar, las corrientes epistemológicas que generaron conocimiento científico, en este punto sobresalen la tradición aristotélica y galileana, dos ideas que difieren cuando tratan de responder la interrogante ¿Qué es ciencia?, estos dos pensamientos se concretizaron en el tiempo y en el espacio, fueron en su momento refutadas, reconstruidas por filósofos y escuelas científicas, convirtiéndose en hitos, que en la actualidad mantienen vigencia en los lugares donde se construye conocimiento científico. En segundo lugar, se presenta un esbozo de las principales corrientes y el desarrollo de las ciencias, en particular el comportamiento de la Geografía frente a las corrientes epistemológicas y en tercer lugar, se señalan aquellas corrientes o enfoques que tienen relevancia para el estudio de la identidad territorial.

Dicho lo anterior, es importante señalar que las corrientes epistemológicas tienen su origen en la necesidad de dar respuestas eficaces a los problemas e intereses del contexto social, económico y político que imperaron en un momento específico de la historia, así mismo éstas se alimentaron por los descubrimientos que acaecieron en el tiempo.

\section{DESARROLLO}

\section{Corrientes epistemológicas que construyen el conocimiento}

Para iniciar, hablar del pensamiento de Aristóteles, es partir del planteamiento que la observación es primacía de la ciencia. Este filósofo sostuvo que toda explicación científica de los fenómenos parte en primera instancia de la observación, también planteó que la investigación tiene dos momentos, uno, la inducción que permite partir de lo particular para establecer generalidades y dos, la deducción que contiene principios para explicar la ocurrencia de los hechos. La filosofía aristotélica resalta el valor de la metafísica y la causa final.

Para la tradición Galileana, la observación no es el principio de la investigación. Esta propone que la explicación de los fenómenos tiene que formularse en término de leyes y que relaciona determinados fenómenos numéricamente, acompañada de hipótesis causales y análisis experimental para generar valor explicativo de la ciencia. Esta tradición considera que la ciencia es funcional y mecanicista (Echano Basaldúa, 1982). Los filósofos que continuaron con el legado de Galileo fueron: Roberto Grosseteste, Roger Bacon, Duns Scoto, Guillenno de Occam y Nicolas de Autrecourt.

La ciencia no es estática, es dinámica en su naturaleza y ha evolucionado en el tiempo. A partir de los planteamientos de Aristóteles y Galileo se gestaron corrientes epistemológicas, las cuales en esencia se fundamentan en ambas tradiciones, sin embargo, por surgir en distintos contextos históricos poseen características particulares.

Con relación a lo anterior, se destacan las corrientes del positivismo, de la hermenéutica, de la teoría crítica y de complementariedad. Es así como estos distintos enfoques de la ciencia generan dos áreas disciplinares bien marcadas: las ciencias naturales y sociales, ambas se enfrentaron y disputaron el hecho de ostentar la verdad sobre los requisitos que debe llevar la explicación de los fenómenos. A continuación, 
se presentan los principales planteamientos y representantes de las ciencias naturales y sociales, así mismo se hará hincapié en la contribución de estos enfoques epistemológicos en el progreso de la Geografía.

\section{El positivismo y la geografía positivista}

El deseo de realizar una ciencia positivista es una aspiración que procede del siglo XVIII. Empecemos por el término "positivo", este se utilizó durante la segunda mitad de esa centuria como contrapuesta al sistema, valorando los datos de la observación frente a los sistemas científicos típicos del racionalismo de la ilustración. Cuando a finales de ese mismo siglo los científicos naturalistas piropeaban la realización de observaciones y defendían que hasta el más pequeño dato reunido a otros era importante para el avance de la ciencia, hicieron gala del espíritu empírico.

El positivismo puede definirse como un método científico y una concepción filosófica del mundo, ambos aspectos están estrechamente ligados entre sí. Como método científico el positivismo es un empirismo inductivo racionalista y anti metafísico. A ello va unida una posición naturalista y un reduccionismo científico en el que las ciencias de la naturaleza se convierten en el modelo de toda cientificidad, lo que, a su vez, deriva de la concepción monista del mundo. Sus principales representes, fueron: Alexander Humboldt, Rasmus Rask, Jacob Grimm, Frederick Tylor, Saint Simon, Auguste Comte, Herbert Spencer y Carlos Marx. Auguste Comte es considerado el padre de este movimiento.

El planteamiento de la corriente positivista se resume en cuatro aspectos fundamentales (Mardones \& Ursua, 1982).

1. El monismo metodológico. En este aspecto se plantea que los objetos de estudio de las ciencias son diversos, sin embargo, existe una unidad de método y homogeneidad doctrinal; en pocas palabras, solo se puede concebir de una única manera aquello que se considere una auténtica explicación científica de los hechos.

2. El canon de las ciencias naturales exactas. El método positivo contiene un canon metodológico fundamentado en la física-matemática, ideal para medir la cientificidad de todas las ciencias.

3. La explicación causal como característica de la explica científica. La explicación de los hechos es causal, esta es acompañada por la búsqueda de leyes generales hipotéticas de la naturaleza que interpretan los fenómenos individuales.

4. El interés dominador del pensamiento positivista. Este aspecto resalta la predicción de los fenómenos. Se traduce en el control y dominio de la naturaleza hasta del mismo hombre como objeto de investigación.

En Geografía, la pretensión de realizar una ciencia positiva apareció tempranamente. En la mitad del siglo XVIII surgieron diversos sistemas geográficos, en los cuales a partir de la aceptación de los principios generales permitieron deducir la configuración orográfica y lacustre de los continentes o regiones poco conocidas; frente a estos, los datos positivos mostraron falsedad de esas especulaciones cartográficas y las hicieron caer en descrédito. De la misma manera, frente a las especulaciones del naturalista Georges 
Leclerc, conde de Buffon ${ }^{1}$, sobre la existencia del continente austral basándose en consideraciones acerca de la formación de los hielos australes por grandes ríos procedentes del polo sur, las expediciones de James Cook², La Perouse ${ }^{3}$ hicieron avanzar a la geografía positivista mediante los descubrimientos y observaciones en mares poco conocidos (Capel, 1981).

Como se sabe, la corriente de la geografía positivista se desarrolló en Europa, sobre todo, en el siglo XVIII, y por eso impregnó fuertemente el pensamiento científico de los primeros maestros de la geografía institucionalizada. A esta influencia se unió también el impacto de la teoría de la evolución de Darwin y de los primeros creadores de la ecología biológica.

El impacto de las ideas positivistas y evolucionistas fue particularmente intenso en la geografía alemana, debido a la relativamente temprana institucionalización de esta ciencia en dicho país. Uno de los precursores de esta corriente fue Friederich Ratzel. Su gran mérito fue haber escrito su obra Antropogeografía. Principios de aplicación de la ciencia geográfica a la Historia, en ella planteó la convergencia entre su concepción orgánica o biogeografía, su preocupación por los problemas de la difusión y migraciones, y la tendencia etnográfica (Capel, 1981).

En los últimos decenios del siglo XIX se atribuyeron cambios importantes en el panorama científico y filosófico europeo y hubo una reacción en contra del positivismo y el naturalismo.

\section{El surgimiento de la hermenéutica}

A finales del siglo XIX e inicio del XX, las concepciones positivistas entraron en crisis, la que se tradujo en el desarrollo de corrientes filosóficas neoidelaistas, neocriticistas o neokantianas, espiritualistas y contingentitas. La crisis del positivismo se reflejó en la crítica del modelo naturalista de cientificidad y en la afirmación de la especificidad de las ciencias humanas, derivada del rechazo del monismo metodológico y de la afirmación correlativa de la antinomia naturaleza-historia. Esto tuvo consecuencias metodológicas importantes, como fue el desplazamiento del objetivo del conocimiento científico desde la explicación a la comprensión y la necesidad de justificar teóricamente una ciencia a partir de lo singular (Capel, 1981). Dicho lo anterior, en este contexto surge la hermenéutica, una corriente anti positivista que rechazó el monismo, la fisicomatemática como canon ideal regulador de toda explicación científica; el afán predictivo y causalista y la reducción de la razón a la razón instrumental. El movimiento hermenéutico considera que el hombre es sujeto de investigación y no un simple objeto como supone el positivismo, así mismo, plantea que el ser humano expresa su interioridad a través de manifestación sensible reflejada en la

\footnotetext{
1 Fue el primero en plantear el principio de la biogeografía mientras examinaba el mundo animal en su Histoire Naturelle, al notar que pese a la similitud de los ambientes las especies que habitaban las distintas regiones eran distintas. Esto se conoció posteriormente como la Ley de Buffon.

2 Fue un navegante, explorador, cartógrafo y capitán de la Marina Real británica. Elaboró mapas detallados de la isla de Terranova antes de realizar tres viajes al Océano Pacífico, durante los cuales estableció el primer contacto registrado de los europeos con la costa oriental de Australia y las islas Hawái, así como la primera circunnavegación de Nueva Zelanda. Realizó once años de navegación por el océano Pacífico, los cuales contribuyeron en gran medida a acrecentar los conocimientos europeos sobre la zona. Muchas islas, como la isla de Pascua y el archipiélago de Hawái, fueron localizadas con certeza por primera vez, y su mayor logro fue la creación de cartografía naval de grandes áreas del Pacífico de gran precisión.

3 Jean François Galaup, conde de La Pérouse. Fue un marino francés, seleccionado por el rey Luis XVI para dirigir una expedición alrededor del mundo, cuyo objetivo era completar los descubrimientos llevados a cabo por James Cook en el océano Pacífico.
} 
conducta, en la colectividad, en sociedad. En esta corriente la comprensión es el principio fundamental de las ciencias.

La comprensión tiene una resonancia psicológica, es una forma de empatía o identificación afectivomental que reactualiza la atmosfera espiritual, sentimientos, motivos, valores, pensamientos, de su sujetoobjeto de estudio. Como se puede apreciar, ciencias como Historia, Antropología, Geografía Humana entre otras, tienen su fundamento filosófico y epistemológico en la hermenéutica (Echano Basaldúa, 1982).

En este enfoque epistemológico el estudio del comportamiento traducido en costumbres, expresiones culturales, sentimientos, cobran importancia en el mundo de la ciencia. La comprensión del significado del comportamiento humano como un hecho social. Sus principales representantes, fueron: Droysen, Dilthey, Simmel y Weber, la escuela de Baden, Windelband, Rickert, Croceyallngles y Collingwood.

En Geografía, el surgimiento del movimiento hermenéutico coincide con el desarrollo de la escuela regional y del paisaje, con la afirmación del carácter ideográfico de la ciencia, con inflexión en la metodológica científica, que acepta facultades como la intuición y desvía el objetivo hacia la comprensión de la realidad compleja (Capel, 1981). Aunque ello suponga una fragmentación de la ciencia geográfica en geografía física y humana. En este sentido surgieron geografos que tratarón de relacionar ambos ramas y proponen que la geografia es una ciencia de sistema y que su objeto "espacio geográfico" es una construcción social y por consiguiente es necesario estudiar la relación entre el ser humano, sociedad y naturaleza o ambiente.

En relación a lo espuesto anteriormente, la geografia humana parte de dos principios fundamentales, en primer lugar, el principio de actividad que contempla que los hechos geográficos, fisicos o humanos, son hechos en perpetua transformación y deben ser estudiados como tales; ello conduce a una perspecva dinámica. El segundo principio es el de conexión que plantea que los hechos de la realidad esta intimamente relacionados entre sí y deben estudiarse en sus multiples conexiones.

\section{Positivismo lógico y la nueva Geografía}

Para Echano (1982), el positivismo lógico o neopositivismo plantea que únicamente los enunciados sometidos a la lógica y a la verificación empírica, pueden ser calificados como científicos, los demás son considerados como absurdos y que la explicación de los hechos tiene su base en el análisis lógico del lenguaje y la verificación empírica. Uno de sus máximos exponentes fue Rudolf Carnap ${ }^{4}$ del Círculo de Viena.

\footnotetext{
4 Filósofo y lógico, uno de los líderes del neopositivismo. Fue un activo miembro del Círculo de Viena. Enseñó filosofía en las Universidades de Viena y de París. Desde 1936, trabaja en los Estados Unidos, es profesor de filosofía de la Universidad de California. Carnap niega el carácter de la filosofía como concepción del mundo y la reduce al "análisis lógico del lenguaje" de la ciencia, basado en los recursos de la lógica matemática. En la concepción de Carnap, los fundamentos teórico-cognoscitivos de dicho análisis representan una unión del empirismo idealista y del convencionalismo en la interpretación de la lógica y de la matemática. La concepción filosófica del neopositivismo se entreteje en los trabajos de Carnap, con las investigaciones sobre teoría de la lógica y el análisis lógico-metodológico de la ciencia.
} 
Carnap planteó que el lenguaje científico ideal es universal, está hecho de símbolos nuevos, neutrales, unívoco. La exactitud y precisión son elementos clave en este planteamiento. Otro elemento importante que señala este filósofo, es el principio de la comprobación y verificación empírica de todas las afirmaciones. Mardones \& Ursua, (1982) mencionan al respecto "Únicamente se tendrá por verdadero y pleno de sentido lo que expresa un estado de cosas objetivo, lo cual equivale a decir, capaz de ser sometido a observación directa y comprobación mediante experimentos".

La filosofía analítica no da paso a la negación de hipótesis. En cambio el filósofo Karl Popper ${ }^{5}$ manifiesta que el conocimiento no es absolutamente seguro y que este tiene el mérito de la duda por consiguiente está sometido a hipótesis conjeturales, a la falsificación. Mardones \& Ursua, (1982), se refiere a esto, cuando comenta que la ciencia no es posesión de la verdad, sino que esta en una constante busquedad crítica de la misma, y que las explicaciones cientificas pueden ser negadas o refutadas, para dar lugar a un nuevo conocimiento.

Para Popper establecer un criterio de demarcación entre la ciencia y la metafísica (y el ser) de manera que sea posible distinguir las proposiciones científicas de aquellas que no los son. Desprecia el inductivismo, para él primero son las teorías porque al pasar de un enunciado singular a un enunciado universal, un solo hecho que no resulte como los demás, puede dar por falsa la teoría, es decir que todas las ciencias tienen que someterse primeramente al método deductivo, debe de existir un esquema lógico basico de teorías, donde el fenómeno que se explicará será un producto de plantemientos hipotéticos iniciales.

\section{El neopisitivismo y las ciencias sociales ¿Que ocurrió con la ciencias sociales y en especial con la Geografía?}

El periodo entre 1030-1940 fue considerado como desicivo en la evolución de las ciencias sociales, porque en esa etapa entraron en crisis muchas ideas desarrolladas a partir del siglo XIX. Esta crisis coincidió con una profunda crisis social y económica.

Las ciencias sociales fueron solicitadas desde diversos frentes en demanda de respuestas eficaces a los problemas como: necesidad de superar la crisis económica del sistema capitalista, que provoca la aparición de la econometría y de la economía positiva; demanda de instrumentos más eficaces de control social, que tuvo efectos inmediatos en la socioogía y en la psicología social (mejora en la técnicas de encuenta sociales, investigaciones sobre actitudes y conflictos); exigencia de la planificación regional y urbana generadas por la misma crisis económica y por la necesidad de atender a la reconstucción de las regiones devastadas por la guerra; luego de la segunda guerra mundial se unió el subdesarrollo suscitado por el proceso de descolonización (Capel, 1981).

5 Fue filósofo y profesor austriaco, aunque más tarde se convirtió en ciudadano británico. Es considerado como uno de los filósofos de la ciencia más importantes del siglo XX. Popper argumentó que una teoría en las ciencias empíricas nunca puede ser probada, pero puede ser falsada, lo que significa que puede y debe ser examinada por experimentos decisivos para distinguir la ciencia de la no-ciencia (es aquella afirmación, creencia o práctica que es presentada como científica y fáctico, pero es incompatible con el método científico. A menudo se caracteriza por el uso de afirmaciones vagas, contradictorias, exageradas o infalsables; la dependencia en el sesgo de confirmación en lugar de pruebas rigurosas de refutación; poca o nula disposición por parte de sus seguidores a aceptar evaluaciones externas de expertos; y en general, la ausencia de procedimientos sistemáticos para el desarrollo racional de teorías). 
Los avances teconológicos generados rapidamente por la primera guerra mundial, permtieron que las ciencias sociales se estimularan para facilitar respuestas más rigurosas y técnicas. Dentro de estos adelantos, estuvieron: las computadoras, nuevos marcos teóricos y concpetuales, tales como la teoría de los sistemas, de la información y la comunicación, entre otros, hicieron el cambio inevitable en los métodos y teorías de estas disciplinas.

La geografía por su parte se vio tambien afectada por la corriente neopositivista, debido a uno de los rasgos que resultaron fue la revolución cuantitativa, de la que surgió la nueva geografía. Esta ciencia se caracterizó por una voluntad explicita positiva que contemplo las explicaciones cientificas y la formulación de leyes generales. El carácter positivista de la nueva geografía fue la aceptación de la unidad profunda de la ciencia, de la posibilidad de transferiri teorías de un campo a otro del conocimiento y el uso de un lenguaje común con otras ciencias, fundamentado en una base matemática (Capel, 1981).

Para la nueva Geografía surgieron nuevos temas de indagación, tales como: localización espacial de la población y actividdaes económicas, que por indudable fue el tema más destacado. Todo giró entorno al análisis del espacio geográfico, la organización espacial, las distribuciones y asociaciones espaciales.

\section{Teoría crítica y Geografía radical}

Durante la decada de 1960, al mismo tiempo que se impuso en las ciencias sociales el optimismo cientifista neopositivista, hubo insastifacción, que cuestionaron los planteamientos de dicha corriente, lo que agudizó aun más la crisis disputa del positivismo y provocó una fuerte crisis en las ciencias sociales. Estas crisis se tradujeron en la proliferación de movimientos críticos o radicales. Al mismo tiempo, surgió la dimensión psicológica y la nueva valoración de la experiencia personal que contribuyó a cuestionar el enfoque positivista; se generó el interés por las corrientes como la fenomenología y el existencialismo. De nuevo la comprensión frente a la explicación (Capel, 1981).

La corriente radical de la Geografía se denominó teoría crítica, concebida por la primera generación de filósofos de la Escuela de Frankfurt en 1923. Fue fundada y dirigida por Horkheimer, junto con Adorno, Marcuse, Fromm, Loventhal, Polloch, estos crearon la teoría crítica de la sociedad. Esta corriente epistemológica se fundamenta en el pensamiento hegeliana-marxista y Freud. Su proposito es analizar la sociedad capitalista occidental y brindar a esta, una propuesta teórica que permita orientarla hacia una sociedad humana, buena y racional. Otro aspecto importante de esta corriente es considerar que la ciencia se asienta en el contexto social, político y económico, por lo tanto la explicación de los fenómenos debe de tomar en cuenta los factores influyentes del contexto social.

Fue a través del descubrimiento de la dimensión personal y subjetiva que la ciencia geográfica cuantitativa comenzó a dar apertura a la nueva geografía radical, la geografía de la percpeción y la del comportamiento, ambas tuvieron un papel fundamental. En este sentido, el marxismo se convirtió en un soporte filósofico y teórico de análisis de los problemas planteados por la geografía. 


\section{Corrientes epistemológicas relevantes para el estudio de la identidad territorial}

Para identificar las corrientes epistemologicas relevantes para el estudio de la identidad territorial, es necesario conceptualizar algunos terminos, como: identidad, identidad territorial y espacio geográfico.

La identidad surge como resultado del proceso de desarrollo, en la medida en que cada comunidad, al evolucionar en el tiempo, va generando costumbres, leyes, productos que forman su cultura; $y$, a la vez, descubrimos que la cultura hace posible el desarrollo.

El espacio geografico como objeto de la Geografía, es entendido como elemento dinámico y cambiante, permite el estudio de los procesos sociales que el ser humano realiza en su quehacer cotidiano para satisfacer sus necesidades; por eso la importancia del conocimiento geográfico y otras disciplinas como la sociología y etnografía para poder entender todos esos procesos sociales que espacialmente se emplazan en el territorio, y qué de manera positiva o negativa afectan los recursos si no se logra un uso adecuado de ellos.

El espacio como territorio se registra a partir de la concepción del espacio como un campo de poder y gestión; es el área comprendida bajo unos límites, donde se llevan a cabo los procesos articulados por las fuerzas políticas, económicas y culturales, a través de un desarrollo social proyectado en el espacio. En él se transforma la naturaleza, a partir del progreso socioeconómico y tecnológico; existe una delimitación del espacio geográfico, y los individuos la ven como su área de pertenencia, es decir, tiene un carácter territorial. Es un espacio finito, de acuerdo con los intereses de quienes lo habitan y utilizan, por lo tanto, tiene un criterio circunstancial.

Moraga G. (2009) señala que la identidad territorial se construye a partir de percepciones, apreciaciones y valoraciones del espacio habitado, que generan un sentido de pertenencia, que lo hacen especial (o único) para sus habitantes, a partir de lo cual van construyendo determinados modos de vida y estrategias que se manifiestan de diferentes formas en el territorio.

El estudio de la identidad territorial traduce aspectos propios de poblaciones y territorios (muchos de ellos subjetivos), ademas que concentra esfuerzos para generar una comprensión y apropiación real de los problemas y dar posibles alternativas de solución a los mismos, en función del buen uso de los recursos naturales.

En este sentido, considero que las corrientes epistemológicas como la hermenéutica, teoría crítica y de complementariedad tiene relevancia para el estudio de la identidad territorila. La primera porque plantea que el ser humano es sujeto-objeto de investigación. Así mismo, plantea que éste expresa su interioridad a través de manifestación reflejada en su conducta, en sociedad, en su cultura, además, la identidad territorial es un elemento determinante que encamina las decisiones de participación social, sobre todo, del sentido de pertenencia al lugar que se habita; la reversión de la segregación socioeconómica y un mejor aprovechamiento de los beneficios que brindan los territorios que ocupan; en tal sentido constituye un elemento clave para la formulación de propuestas encaminadas a democratizar la gestión del espacio 
habitable. Como se puede apreciar tiene relevancia porque está relacionado con el ser humano y la manera de cómo se relaciona con su medio geográfico.

La teoría crítica porque brinda aspectos relacionados con el quehacer de las ciencia sociales, asi mismo plantea que la razón mantiene una relativa autonomía respecto a los hechos, que la sociedad es subjetiva, pero también es objetiva en razón a su estructura y que la objetividad se alcanza con el método crítico. Moraga, (2009) citando a Claval, (2002), hace referencia que desde la corriente humana de la Geografía es posible entender las culturas a partir de los territorios, así como la percepción que se genera de los mismos a partir de la vivencia e interacción de sus habitantes, al identificar aspectos de pertenencia en una población heterogénea, determinado por la lógica de cohesión, se pueden crear estrategias orientadas al uso adecuado y sostenible de los recursos en las comunidades.

La geografía cultural es una rama de la Geografia Social. Fue impulsada por Sauer, en Estados Unidos en los años '50 y '60. Estudia los aspectos simbólicos, discursivos e identitarios del espacio, el lugar como ámbito de poder (territorialidad) y donde se gestan los significados culturales (Cuadra, 2014).

Para hacer referencia a la geografía ambiental se debe partir de tres condiciones generales: en primer lugar, es el saber geográfico. La concepción del ser humano acerca de su mundo que ha cambiado en el transcurso de las épocas históricas, variabilidad que se diversifica en los diferentes enfoques. En segundo lugar, que la cuestión ambiental surge en la actualidad, como efecto de la superación de los límites de recuperación de las bases naturales de las sociedades, efecto de múltiples problemas tecnológicos, ideológicos, políticos y sociales involucrados en la relación de la humanidad con la naturaleza, que se extiende a dintintas escala geográfica.

En tercer lugar, que la geografía ambiental no es una "nueva disciplina", sino un campo estratégico que funde varias subdisciplinas e, incluso, se auxilia de las "ciencias ambientales" para abordar problemáticas inherentes a los sistemas naturales, sociales y de ideas, involucrados en los múltiples y diversos problemas que constituyen la cuestión ambiental. Esto permite deducir la importancia de la ciencia geográfica dentro de las ciencias ambientales (Cervantes Ramirez, Franco Gonzáles, \& Juárez Gutiérrez, 2014).

\section{CONCLUSIONES Y/O RECOMENDACIONES}

En fin, con la revisión bibliográfica se logró apreciar que el desarrollo del pensamiento filosófico de la ciencia ha estado marcado por distintos puntos de vista o corrientes. Es increíble ver cómo las corrientes tradicionales Aristotélicas y Galileana evolucionaron en el tiempo y espacio.

El vaivén de las ciencias naturales y sociales, una lucha constante de poder entre lo positivo y crítico, lo objetivo y subjetivo. Pero este vaivén de las ciencias nos indica que el conocimiento científico es dinámico y evoluciona como respuesta a las demandas sociales, políticas y económicas que se generan en los contextos. La contienda continúa en la actualidad y se manifiesta principalmente en los distintos espacios que generan conocimiento como es el caso de universidades e institutos de investigación. 
La geografía como una ciencia social no ha escapado a esta realidad cambiante. Cada enfoque filosófico ha estimulado sus trasformaciones y por lo tanto su desarrollo como ciencia. Su propio objeto de estudio ha sido enriquecido por los aportes teóricos y conceptuales del positivismo y neopositivismo, de la teoría crítica, con este último enfoque, la geografía emprende un nuevo rumbo que es el pensamiento crítico o radical ante las problemáticas de orden espacial y social.

Indudablemente, las corrientes epistemológicas que tienen relevancia en el estudio de la identidad territorial son: la hermenéutica, teoría crítica y por supuesto hoy se habla de la complementariedad de las ciencias, dado que el estudio de la identidad territorial y ambiente natural requiere de un enfoque inter y multidisciplinario, desde el enfoque de la geografía cultural y ambiental.

Para enriquecer aún más el conocimiento acerca del estudio de identidad territorial se recomienda continuar con la revisión bibliográfica y el análisis documental, dado que permitirá conocer más aportes teóricos relacionados con el tema.

\section{REFERENCIAS BIBLIOGRÁFICAS}

Capel, H. (1981). Filosofía y ciencia en la Geografía contemporánea. Barcelona: Ediciones del Serbal.

Carrasco, O. V. (2009). Cómo escribir artículos de revisión. Revista Médica La Paz, 65.

Cervantes Ramirez, M. C., Franco Gonzáles, A. M., \& Juárez Gutiérrez, M. d. (2014). Geografía Ambiental de México. Distrito Federal: Universidad Nacional Autónoma de México.

Claval, P. (2002). Los fundamentos actuales de la geografía cultural. A.G.E, 22.

Cuadra, D. E. (2014). LOS ENFOQUES DE LA GEOGRAFÍA EN SU EVOLUCIÓN COMO CIENCIA . Revista Geográfica Digital, 6.

Echano Basaldúa, J. (1982). MARDONES, J.-M., y URSUA, N. : Filosofía de las ciencias humanas y sociales. Logos. Anales del Seminario de Metafísica.

Mardones, J., \& Ursua, N. (1982). Folosofia de las ciencias humanas y sociales. Barcelona: Graficas Ampurias.

Moraga López, G. (2009). Geografía Cultural e Identidad Territorial: el caso de la comunidad de Cabuya, distrito de Cóbano, Puntarenas, 2009. Revista geográfica de America Central, 131.

Ramírez, A. V. (21 de mayo de 2009). La teoría del conocimiento en investigación científica: una visión actual. Anales de la facultad de Medicina, http://www.scielo.org.pe/pdf/afm/v70n3/a11v70n3.pdf. Obtenido de Scielo Perú. 